\title{
Complementary and alternative medicine use among women at increased genetic risk of breast and ovarian cancer Christine M Mueller*1, Phuong L Mai ${ }^{1}$, Jaime Bucher ${ }^{2}$, June A Peters ${ }^{1}$, Jennifer T Loud ${ }^{1}$ and Mark H Greene ${ }^{1}$
}

Address: ${ }^{1}$ Clinical Genetics Branch, Division of Cancer Epidemiology and Genetics, National Cancer Institute, National Institutes of Health, Bethesda, Maryland, USA and ${ }^{2}$ College of Medicine, University of Toledo, Toledo, Ohio, USA

Email: Christine M Mueller* - muellerc@mail.nih.gov; Phuong L Mai - maip@mail.nih.gov; Jaime Bucher - jaime.bucher@utoledo.edu; June A Peters - petersju@mail.nih.gov; Jennifer T Loud - loudj@mail.nih.gov; Mark H Greene - greenem@mail.nih.gov

* Corresponding author

Published: 30 April 2008

BMC Complementary and Alternative Medicine 2008, 8: 17 doi:10.1 186/1472-6882-8- 17

This article is available from: http://www.biomedcentral.com/1472-6882/8/17

(C) 2008 Mueller et al; licensee BioMed Central Ltd.

This is an Open Access article distributed under the terms of the Creative Commons Attribution License (http://creativecommons.org/licenses/by/2.0), which permits unrestricted use, distribution, and reproduction in any medium, provided the original work is properly cited.
Received: 28 January 2008

Accepted: 30 April 2008

\begin{abstract}
Background: Complementary and alternative medicine (CAM) use is well documented among breast cancer patients and survivors, but little evidence is available to describe rates and patterns of use among women at increased genetic risk of breast cancer.

Methods: A pre-visit telephone interview was conducted to ascertain CAM use among the BRCA mutation carriers enrolled in a high-risk breast cancer screening study. Participants were asked to report on their use of thirteen therapies within the year prior to enrollment into the study. Logistic regression was used to evaluate the association between various factors and CAM use in this population.
\end{abstract}

Results: Among the 164 BRCAI or BRCA2 mutation-positive (BRCA+) women in this analysis, $78 \%$ reported CAM use, with prayer and lifestyle diet being the two most commonly reported modalities. Many subjects used multiple CAM therapies, with 34\% reporting use of three or more modalities. The most commonly used modalities were mind-body therapies and biologically-based practices, $61.6 \%$ and $51.8 \%$, respectively. High-risk women were more likely to use CAM if they were older, more educated, more worried about ovarian cancer risk, or had a previous cancer diagnosis.

Conclusion: This study suggests that the prevalence of CAM use is high among BRCA mutation carriers, with frequency of use comparable to that of breast cancer patients and survivors. Given the high prevalence of CAM use in our subjects, especially biologically-based therapies including herbal supplements, whose safety and efficacy in relation to cancer risk are unknown, our study suggests that future research is necessary to clarify these risks, and that it is important for providers to inquire about and to discuss the pros and cons of CAM use with their BRCA+ patients.

\section{Background}

Complementary and alternative medicine (CAM) includes a collection of therapies, practices, and products that are not considered to be part of conventional medical practice [1]. The National Center for Complementary and Alternative Medicine (NCCAM) divides CAM therapies 
into five domains, recognizing that overlap exists. They include (with examples): biologically-based practices (herbs, diets), mind-body medicine (prayer, meditation, yoga), energy healing (Reiki, therapeutic touch), manipulative and body based practices (chiropractic care, massage), and whole medical systems (traditional Chinese medicine, Ayurveda). Use of CAM escalated in the United States during the 1990's, with an estimated $\$ 36$ to $\$ 47$ billion spent on these modalities in 1997 alone. In addition, several studies have shown that CAM is most commonly used in conjunction with conventional medical interventions $[2,3]$.

The 2002 National Health Interview Survey (NHIS) of 31,044 US adults, of whom more than 17,000 were women, provides the most recent CAM use data for the general population. In that survey, CAM use was greater among women than men, with sixty-nine percent of women reporting CAM use within the 12 months prior to survey completion. Prayer was the most commonly used modality. When prayer for health reasons was excluded, the prevalence of CAM use among women decreased to $40 \%$. Higher levels of CAM use were associated with increased education, higher income, and poorer health status. In general, women between 50-59 years of age comprised the sub-group with the highest rates of use for most CAM modalities, except for prayer, which continued to increase with advancing age $[2,4]$.

Several studies have shown that cancer patients and survivors are more likely to use CAM than unaffected individuals in the general population, with up to $83 \%$ reporting CAM use [5-8]. Furthermore, women with a current or previous diagnosis of breast cancer have been reported to use CAM more frequently than individuals diagnosed with other cancers [9]. Self-reported motivations for CAM use among recently-diagnosed patients include desires to improve quality of life, enhance immune function, and actively participate in self-care $[6,10]$; higher levels of anxiety and depression have been associated with higher rates of CAM use among cancer patients and survivors [11-13].

While there is an extensive literature regarding CAM use among breast cancer patients, much less is known about its use among women at high genetic risk of developing breast cancer. In a previous study of CAM use within a cohort of women undergoing genetic counseling and testing for germline mutations in BRCA1/2 genes, cancer survivors reported significantly more CAM use than unaffected women [14]. A follow-up to that study showed that $B R C A+$ women were more likely to be using CAM one year after disclosure of their genetic test result, compared with usage rates prior to genetic testing [15].
Women at increased genetic risk of breast and ovarian cancer face difficult decisions when choosing the optimal strategy to manage their cancer risk. Their decisions are often based on personal beliefs, attitudes, and experiences, as expressed by their cancer risk perception and worry [16-20]. Furthermore, high-risk women who choose salpingo-oophorectomy as an ovarian cancer riskreducing option must also face medical decisions regarding the management of postmenopausal symptoms related to surgical menopause. For the general population and breast cancer survivors, this often includes taking herbal supplements, such as soy products, whose safety and efficacy are unproven $[21,22]$. With the increased prevalence of CAM usage in the general population, it is important to identify patterns of use among high-risk women to promote informed medical decision-making and to improve the quality of care that these patients receive. In this study, we comprehensively described and analyzed CAM use in women with known BRCA1 or BRCA2 mutations enrolled in a high-risk breast cancer screening study.

\section{Methods}

The Clinical Genetics Branch Breast Imaging Screening Study in Women at High Genetic Risk of Breast Cancer: Annual Follow-up Study (NCI protocol 01-C-0009) is aimed at evaluating breast cancer screening modalities among women who are genetically predisposed to developing breast cancer. The study consists of four consecutive annual evaluations including mammogram, breast MRI, clinical breast examination, transvaginal ultrasound, CA125 and breast ductal lavage. As part of this protocol, participants were asked to complete a self-administered questionnaire and a telephone questionnaire prior to their first study visit. Participants were eligible for the study if they were between 25 and 56 years of age, had a known BRCA1 or BRCA2 mutation, or were first- or second-degree relatives of a known BRCA mutation-positive family member. Women younger than 25 , but within 5 years of the age at diagnosis of the youngest family member with a hereditary breast-ovarian cancer syndrome-associated tumor, were also eligible.

Exclusion criteria included pregnancy or lactation within 6 months of enrollment, abnormal CA- 125 level, bilateral breast cancer, previous bilateral mastectomy or bilateral radiation therapy, weight over $136 \mathrm{~kg}$, or allergy to gadolinium. Individuals with a personal history of ductal carcinoma in situ (DCIS), Stage I, or Stage IIA breast cancer were eligible provided that at least 6 months had elapsed since completing primary therapy (surgery, radiation, or chemotherapy). Other exclusion criteria included a personal history of ovarian cancer (any stage); breast cancer (stage IIB or higher); DCIS, Stage I or Stage IIA breast cancer with a relapse after primary treatment; or any other 
invasive cancer except non-melanoma skin cancer or cervical carcinoma in situ, unless relapse-free for 5 years prior to the time of enrollment. Informed consent was obtained from all participants, and a total of 200 women were enrolled onto 01-C-0009 between June 2002 and February 2007. A secondary study objective of the study was to determine the rates of CAM use in high-risk women enrolled in this breast cancer screening study related to various participant characteristics and screening behaviors. Of the participants in the Breast Imaging Screening Study, 164 women who know their BRCA+ status and completed their CAM questionnaire were included in this analysis.

\section{Demographics}

Demographic information, including age, race (white, non-white), marital status (single, married/steady relationship, separated/divorced), number of children $(0$, $\geq 1$ ), education (high school or less, college and beyond), and personal cancer history, was obtained through the pre-visit questionnaires.

\section{Complementary and Alternative Medicine Use}

Complementary and alternative medicine data were obtained through the pre-visit telephone interview, which was completed prior to the first study visit. Participants were asked to answer "yes" or "no" to the use of each of thirteen CAM therapies (acupuncture, meditation, relaxation techniques, yoga, massage therapy, imagery, spiritual healing or prayer, lifestyle diet, herbal medicine, homeopathic treatment, energy healing, biofeedback, and hypnosis) within the previous year. No standard examples of these modalities were provided by the interviewer, and no further details regarding frequency, duration, or purpose of use were elicited. Participants were also given the opportunity to report the use of additional therapies. For data analysis purposes, the individual CAM therapies were further grouped by the defined NCCAM domains: biologically-based practices (herbal medicine, lifestyle diet); mind-body medicine (spiritual healing or prayer, meditation, yoga or tai chi, relaxation techniques, biofeedback, imagery, hypnosis); energy healing, manipulative and body-based practices (massage therapy); and alternative medical systems (homeopathic treatment, acupuncture).

\section{Perceived Cancer Risk}

Perceived breast and ovarian cancer risk was assessed for each cancer with the survey question: "In your opinion, compared to other women your age, what are your chances of getting breast/ovarian cancer in your lifetime?" Participants responded with categorical options: "Much less," "A little less," "About the same," "A little more" or "Much more," coded on a 1-5 scale. For the current analysis, responses were dichotomized to average or below average (codes 1-3) and above average (codes 4,5) risk perceptions [16]. Individuals who chose not to answer the question were excluded from the cancer risk perception analysis.

\section{Cancer Worry}

Cancer worry was determined using three validated questions from the Lerman Cancer Worry Scale [23,24], relating to frequency of thoughts of getting either breast or ovarian cancer and their impact on mood and daily activities: "During the past month, how often have you thought about your own chances of getting breast/ovarian cancer; how often have thoughts about your chances of getting breast/ovarian cancer affected your mood; how often have thoughts about breast/ovarian cancer affected your ability to perform your daily activities?" Participants responded with categorical options: "Not at all or Rarely," "Sometimes," "Often," "A lot," coded on a 1-4 scale. The average score was calculated for each cancer to determine their level of cancer worry. Individuals who chose not to answer the questions were excluded from the cancer worry analysis.

\section{Depression}

Depression was measured using the 20 question Center for Epidemiologic Studies Depression (CES-D) Scale, a short self-report instrument designed to measure depressive symptoms in the general population, emphasizing depressed mood, the major affective component of depression [25]. Each question was scored from 0 to 3 based on the frequency of occurrence within the past week, with responses then summed, for a total possible score ranging from 0 to 60 . We grouped the scores into $<16$ and $\geq 16$, since a score of $\geq 16$ is indicative of clinical depression [25]. For individuals with four or fewer missing responses, a person-mean imputation technique based on the existing responses was utilized to develop a total score. The mean value of the individual's completed items is substituted into the missing items to calculate a total CES-D score as previously described [26]. Individuals with $>4$ missing responses were excluded from the depression analysis.

\section{Screening Behavior}

Participants provided detailed information on how frequently they performed cancer screening activities before study enrollment. For mammography and transvaginal ultrasound (TVU), we dichotomized participants as either "following" or "not following" appropriate screening guidelines (monthly breast self exam (BSE), annual mammography, annual TVU) based on self-reported screening frequency $[16,27]$. The date of mutation status disclosure was defined as the time from which appropriate screening practices should have been followed. Individuals who screened more frequently than recommended guidelines were considered to be following guidelines. Women who 
reported having bilateral oophorectomy $(\mathrm{n}=69)$ were excluded from the TVU data analysis.

\section{Statistical Analysis}

For comparison between CAM users and non-users, Chisquare and Fisher's Exact tests were used as appropriate for categorical variables, and Wilcoxon rank-sum test was used for continuous variables. We used logistic regression to evaluate the association between various factors and CAM use. Factors with a p-value of $\leq 0.20$ in the univariate analysis were included in the final multivariate logistic regression model. Factors with a two-tailed p-value of $\leq 0.05$ in the multivariate model were considered to be statistically significant. All statistical analyses were conducted using SPSS 15.0.

\section{Results}

Of $165 B R C A+$ women who knew their mutation status prior to enrolling in the Breast Imaging Screening Study, 164 answered the pre-visit telephone CAM questionnaire and were included in this analysis. The cohort was highlyeducated (92.7\% attending college); predominately white (97.6\%); most were married (72\%); over half reported having children (54.3\%); and $17.7 \%$ had a personal history of cancer prior to study entry.

The overall CAM use rate in our BRCA+ population was $78 \%(128 / 164)$, with an average of 2.3 CAM therapies used per person. Thirty-four percent of the cohort reported having used three or more CAM therapies within the past year. As shown in Figure 1A, only 15 (9.1\%) $B R C A+$ women reported prayer as the sole CAM therapy used. If spiritual healing/prayer are excluded, $68.9 \%$ of participants had used CAM in the previous year. Spiritual healing/prayer and lifestyle diet were the most commonly reported modalities ( $48.8 \%$ and $48.2 \%$, respectively). Figure $1 \mathrm{~B}$ shows CAM use grouped by NCCAM domains: biologically-based practices (herbal medicine, lifestyle diet); mind-body medicine (spiritual healing or prayer, meditation, yoga or tai chi, relaxation techniques, biofeedback, imagery, hypnosis); energy healing, manipulative and body-based practices (massage therapy); and alternative medical systems (homeopathic treatment, acupuncture). Mind-body therapies and biologically-based practices were the most commonly used domains $(61.6 \%$ and $51.8 \%$, respectively). However, if prayer is excluded from the computations, biologically-based practices become the most prevalent modality.

There were significant positive associations between CAM use and several demographic, health-related, and psychosocial variables (Tables 1 and 2$)$. Higher education ( $\mathrm{p}=$ $0.015)$, a previous cancer diagnosis $(\mathrm{p}=0.006)$, and older age $(\mathrm{p}=0.007)$ were all associated with increased CAM use. Although the overall scores were not high $($ median $=$
1.33), higher ovarian cancer worry scores were statistically significantly associated with increased CAM use ( $\mathrm{p}=$ 0.011 ) (Table 2). These findings did not change when the ovarian cancer worry analysis was restricted to the 95 women with intact ovaries (data not shown); therefore, all women were included in the multivariate regression analysis. Among the demographic indicators, race $(\mathrm{p}=1.00)$, number of children $(\mathrm{p}=0.52)$, and marital status $(\mathrm{p}=$ 0.91 ) were not associated with CAM use, and neither were the health-related behaviors of breast or ovarian cancer screening, nor the psychosocial variables depression, perceived cancer risk, and breast cancer worry.

Factors with $\mathrm{p} \leq 0.20$ (age, education, previous cancer diagnosis, ovarian cancer worry, breast cancer worry, and BSE) were included in the multivariate logistic regression model. Odds ratios and 95\% confidence intervals are presented in Table 3. Age, higher education, previous cancer diagnosis, and increased ovarian cancer worry remained statistically significantly associated with CAM use ( $\mathrm{p} \leq$ $0.05)$. Previous cancer diagnosis showed the strongest association with increased CAM use; women with any previous cancer were 17 times more likely to use CAM than unaffected women. Multivariate analysis showed a weak, yet statistically significant, inverse association between frequency of BSE and CAM use; women who did BSE less than once a month were more likely to use CAM.

Since a previous cancer diagnosis was highly-associated with CAM use in our population, a finding previouslyreported in the general population literature, we repeated our analyses after excluding the women with a previous cancer diagnosis. The overall patterns of the CAM therapies used remained unchanged (data not shown), with the exception of lifestyle diet being more commonly used than spiritual healing/prayer ( $47.4 \%$ and $44.4 \%$, respectively) compared with $48.2 \%$ and $48.8 \%$, respectively in the previous analysis. When spiritual healing/prayer was excluded, overall CAM use was $65.2 \%$ compared to $68.9 \%$ when all $B R C A+$ women were included in the analysis.

Age, education, previous cancer diagnosis, ovarian cancer worry, breast cancer worry, and BSE all had $\mathrm{p} \leq 0.20$ in the univariate analyses again and were included in the multivariate logistic regression model. Ovarian cancer risk perception was also included in the multivariate analyses with $\mathrm{p}=0.15$. As was the case with the previous analysis, age, higher education, ovarian cancer worry, and BSE remained statistically significantly associated with CAM use ( $\mathrm{p} \leq 0.05)$, with similar odds ratios. Ovarian cancer risk perception was no longer statistically significant.

\section{Discussion}

Our study provides the largest, most comprehensive descriptive analysis of CAM use among BRCA mutation 
A. Patterns of CAM Use In BRCA+ Women by Individual CAM Therapy

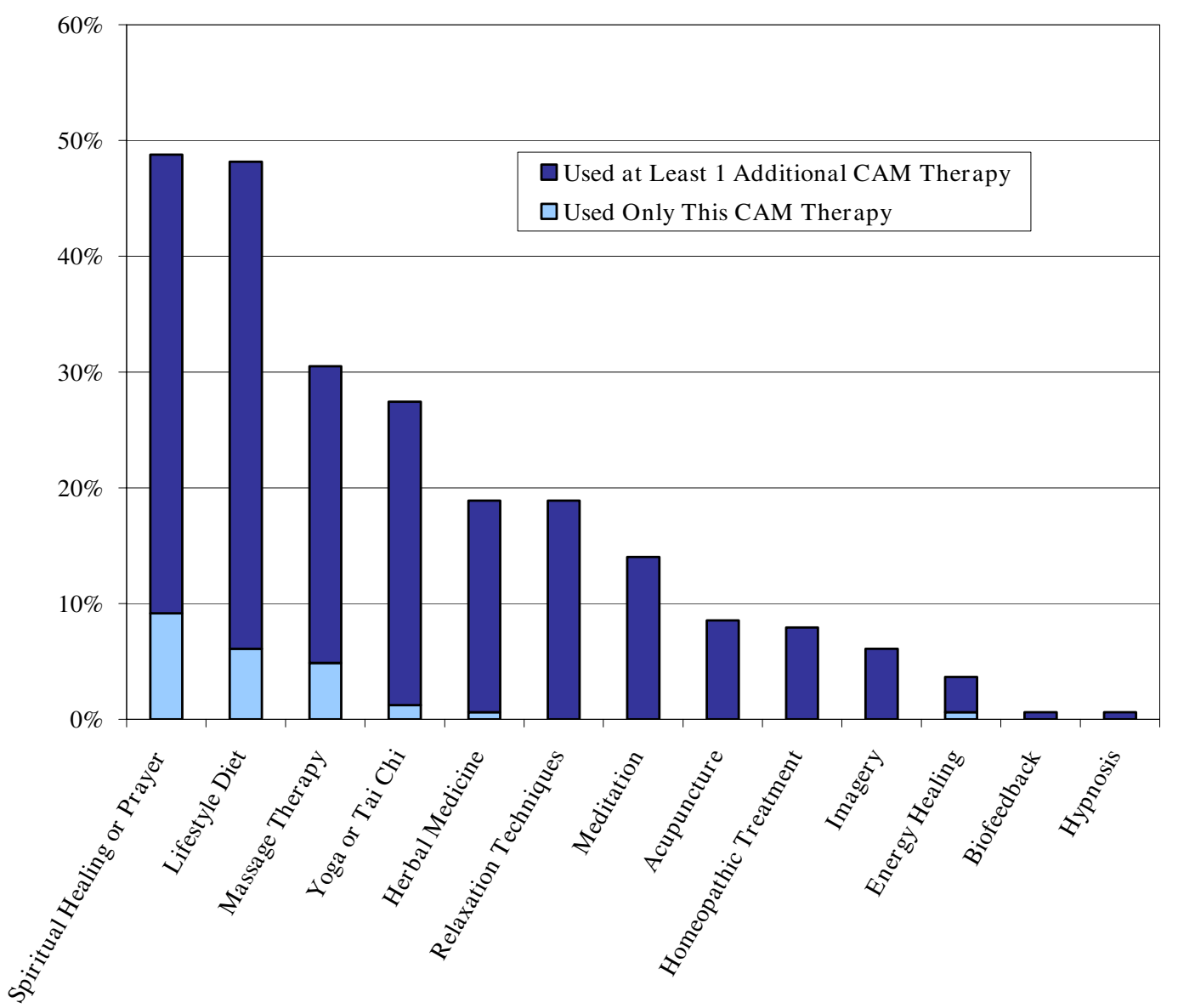

B. CAM Use by Domain

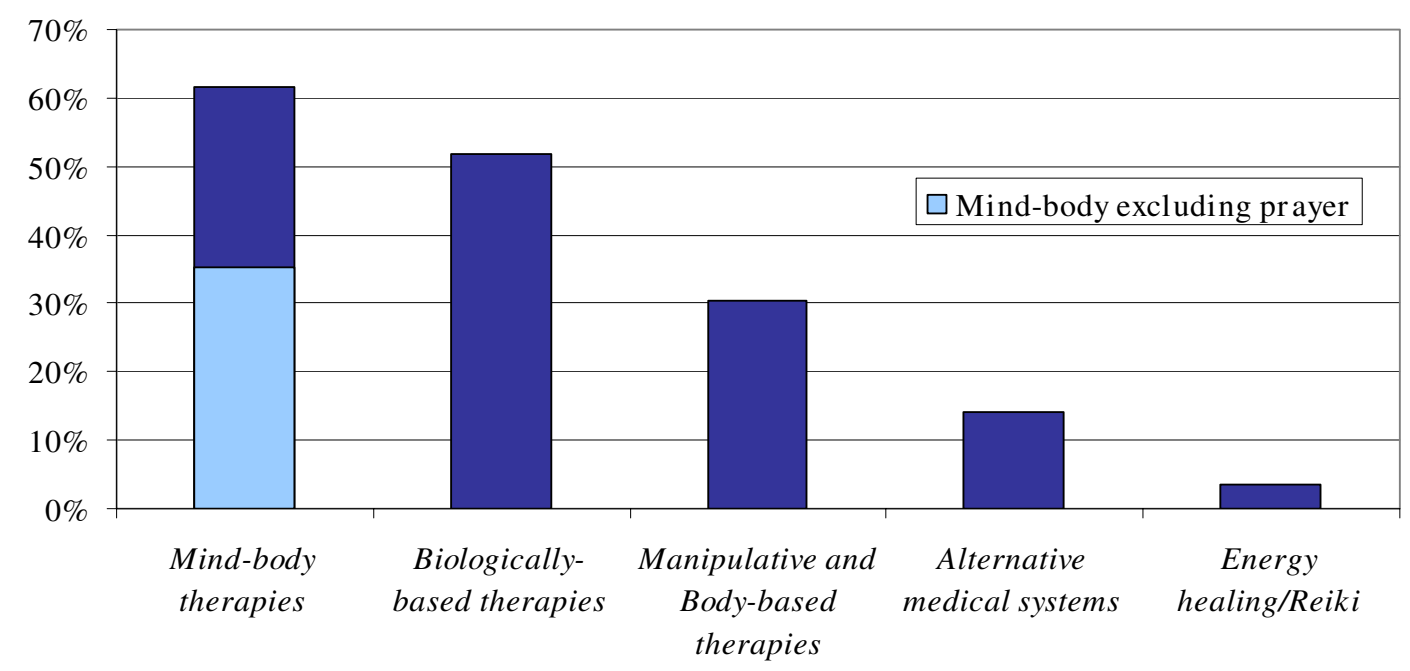

Figure I

Patterns of CAM Use in BRCA+ Women. 
Table I: Associations Between CAM Use and Demographic, Psychosocial and Health-related Categorical Predictor Variables in BRCA+ Women

\begin{tabular}{|c|c|c|c|}
\hline Variable & CAM User (\%) & CAM Nonuser (\%) & $\mathbf{p}$ \\
\hline \multicolumn{4}{|l|}{ Education } \\
\hline High School or Less & $6(4.7)$ & $6(16.7)$ & 0.015 \\
\hline Some College and Beyond & $122(95.3)$ & $30(83.3)$ & \\
\hline \multicolumn{4}{|l|}{ Race/Ethnicity } \\
\hline White & $125(97.7)$ & $35(97.2)$ & 1.00 \\
\hline Non-White & $3(3.3)$ & $\mathrm{I}(2.8)$ & \\
\hline \multicolumn{4}{|l|}{ Children } \\
\hline 0 & $56(43.8)$ & $17(50.0)$ & 0.52 \\
\hline$\geq 1$ & $72(56.3)$ & $17(50.0)$ & \\
\hline \multicolumn{4}{|l|}{ Marital Status } \\
\hline Single & $27(2 \mid .3)$ & $7(19.4)$ & 0.91 \\
\hline Married/Steady Partner & $91(71.7)$ & $27(75.0)$ & \\
\hline Separated/Divorced & $9(7.1)$ & $2(5.6)$ & \\
\hline \multicolumn{4}{|l|}{ Previous Cancer History* } \\
\hline Yes & $28(21.9)$ & I (2.8) & 0.006 \\
\hline No & $100(78.1)$ & $35(97.2)$ & \\
\hline \multicolumn{4}{|l|}{ CES-D Score } \\
\hline Depressed & $25(19.7)$ & $6(16.7)$ & 0.68 \\
\hline Not Depressed & $102(80.3)$ & $30(83.3)$ & \\
\hline \multicolumn{4}{|l|}{ Breast Cancer Risk Perception } \\
\hline Average or Below Average Risk & $3(2.4)$ & I (2.9) & 1.00 \\
\hline Above Average Risk & $121(97.6)$ & $33(97.1)$ & \\
\hline \multicolumn{4}{|l|}{ Ovarian Cancer Risk Perception } \\
\hline Average or Below Average Risk & $34(27.4)$ & II (32.4) & 0.57 \\
\hline Above Average Risk & $90(72.6)$ & $23(67.6)$ & \\
\hline \multicolumn{4}{|l|}{ Breast Self Exam } \\
\hline Less than Once per Month & $58(46.0)$ & II (3I.4) & 0.12 \\
\hline At Least Once per Month & $68(54.0)$ & $24(68.6)$ & \\
\hline \multicolumn{4}{|l|}{ Mammography } \\
\hline Not Following Guidelines & $25(19.5)$ & $10(27.8)$ & 0.29 \\
\hline Following Guidelines & $103(80.5)$ & $26(72.2)$ & \\
\hline \multicolumn{4}{|c|}{ Transvaginal Ultrasound *limited to 95 women with ovaries } \\
\hline Not Following Guidelines & $22(29.3)$ & $4(20.0)$ & 0.57 \\
\hline Following Guidelines & $53(70.7)$ & $16(80.0)$ & \\
\hline
\end{tabular}

* Number of cancers by site: breast cancer $=23$ (two with multiple primaries I = melanoma, I = basal cell carcinoma), ovarian cancer = I, melanoma $=\mathrm{I}$, thyroid $=\mathrm{I}$, vaginal clear cell adenocarcinoma $=\mathrm{I}$, basal cell carcinoma $=2$

carriers who are at increased genetic risk of breast and ovarian cancer, and who had undergone risk assessment and counseling prior to enrollment. Compared with data from women in the general population [4], the high-risk women enrolled in our cohort were more likely to use CAM (78\% vs. 69\%), a rate which is comparable to that reported for breast cancer patients and survivors [5-8]. When prayer is excluded, only $40 \%$ of women in the general population used CAM, compared with $68.9 \%$ of the BRCA+ women in our study [4]. Therefore, although prayer was also the most commonly used CAM modality in our population, in contrast to the pattern of use observed in the general population, it was commonly used in addition to other modalities.

CAM use in our cohort was associated with older age, higher education level, and higher levels of ovarian cancer worry. A previous cancer diagnosis showed the strongest association with increased CAM use in our BRCA+ population, consistent with reports based on general popula-

Table 2: CAM Use and Demographic, Psychosocial and Health-related Continuous Predictor Variables in BRCA+ Women

\begin{tabular}{|c|c|c|c|}
\hline & CAM User Median (Range) & CAM Nonuser Median (Range) & $\mathbf{p}$ \\
\hline Age & $41.0(22.0-55.0)$ & $35.0(23.0-55.0)$ & 0.007 \\
\hline Breast Cancer Worry & $1.67(1.00-3.67)$ & $1.33(1.00-2.67)$ & 0.18 \\
\hline Ovarian Cancer Worry & $1.33(1.00-3.67)$ & $1.00(1.00-2.33)$ & 0.011 \\
\hline
\end{tabular}


Table 3: Multivariate Associations Between CAM Use and Predictor Variables in BRCA+ Women

\begin{tabular}{lcc}
\hline & Odds Ratio (95\% CI) & P \\
\hline Education & $10.3(2.0-53.8)$ & .006 \\
Personal Cancer History & $17.1(1.5-191.4)$ & .021 \\
Breast Self Exam & $0.3(0.1-0.8)$ & .017 \\
Age & $1.1(1.0-1.1)$ & .016 \\
Breast Cancer Worry & $1.0(0.4-2.4)$ & .958 \\
Ovarian Cancer Worry & $7.9(1.7-38.1)$ & .009 \\
\hline
\end{tabular}

tion samples [5-8]. In a previous report on high-risk women, cancer survivors reported more CAM use than unaffected women; however, all univariate and multivariate analyses in that study were conducted separately for these groups [14]. Since our sample included only a small number of participants with a previous cancer diagnosis, analyzing CAM use stratified by previous cancer history would have had limited statistical power. As an alternative, we performed our analyses excluding the women with a previous cancer diagnosis, and found no meaningful differences in results.

Previous studies have reported conflicting evidence for the association between age and CAM use both in the general population and in breast cancer patients and survivors $[2,6,28,29]$, while other studies have shown no association between age and CAM use $[15,30]$. Some of this discordance may be due to the manner in which age is dichotomized in different studies. The age group with the most extensive CAM use in the general population was between 50-59 years [2]. In our study, CAM use increased with age, but the oldest subject in our cohort was only 55 . Thus, we were not able to evaluate CAM use in older women.

This study showed no association between CAM use and perceived cancer risk (breast and ovarian) or breast cancer worry. CAM users tended to be more worried about ovarian cancer, but overall the reported levels of breast and ovarian cancer worry were low. Higher rates of CAM use have been associated with increased levels of depression in cancer survivors and individuals with chronic illness in some studies $[2,13,31]$, a finding we did not corroborate. These results may be related to the homogeneity of our study participants, all of whom have been informed of their increased cancer risk through genetic counseling, knew themselves to be BRCA mutation-positive; have chosen breast cancer screening rather than prophylactic mastectomy to manage their risk; and, for the majority, have not developed a BRCA-related malignancy. These characteristics, in the aggregate, may yield a group of women which is less worried about developing cancer than the more heterogeneous groups of high-risk women who have been studied previously.

Prior reports have suggested that high-risk women report better health maintenance practices than do women from the general population $[14,16]$. Therefore, we evaluated potential associations between CAM use and cancer screening practices for breast and ovarian cancer, covariates which have not been previously examined. The majority of our study participants followed recommended screening guidelines. The only significant, although weak, association that we found was that women who did BSE less than once a month were more likely to use CAM.

Limitations to our study include the reliance on selfreported data regarding CAM use. Participants were not provided examples for each of the thirteen modalities, and thus may have interpreted modalities such as lifestyle diet differently. Furthermore, we did not collect data on duration and frequency of use for each CAM, nor did we elicit information regarding specific motives for CAM use such as cancer prevention. Such data might have provided further insight into the lifestyle choices that our subjects have made in their efforts to prevent breast and ovarian cancer, and cope with the increased stress related to knowing that they are at high risk of malignancy. Another limitation of the study is a relatively small sample size both overall and of cancer survivors, providing for minimal statistical power for some analyses. Finally, our study population should not be considered representative of all $B R C A$ mutation carriers, since it was comprised of research volunteers who were almost exclusively white and highly-educated, both characteristics that are associated with higher rates of CAM use in the general population.

\section{Conclusion}

We have documented that the rates of CAM use in our cohort of BRCA+ women, most of whom have never developed cancer, are roughly equivalent to the rates reported for women being treated for, or having survived, breast cancer, and seem to be higher than the rates reported for women from the general population. Further investigation will be required to better understand the intensity of, and motivations for, CAM use, as well as the relationships between CAM use, conventional screening practices, and cancer risk and worry in BRCA+ women. Data from a more representative sample of all mutation carriers would likely be very informative as well. However, given the high prevalence of CAM use in our subjects, especially the predominance of biologically-based therapies, which might pose a greater risk of unanticipated adverse effects, our study suggests that it is important for providers to inquire about, and to discuss the pros and cons of CAM use, with their BRCA+ patients [32]. 


\section{Abbreviations}

Complementary and alternative medicine: CAM; National Center for Complementary and Alternative Medicine: NCCAM; National Health Interview Survey: NHIS; ductal carcinoma in situ: DCIS; Center for Epidemiologic Studies Depression: CES-D; transvaginal ultrasound: TVU.

\section{Competing interests}

The authors declare that they have no competing interests.

\section{Authors' contributions}

CMM, PLM, and JB performed the statistical analysis. CM was the primary author and wrote the final manuscript. All authors helped to conceive the study, participated in its design and coordination, and contributed to drafting the manuscript. All authors read and approved the final manuscript.

\section{Acknowledgements}

This work was supported by funding from the National Cancer Institute Intramural Research Program. The protocol patients described herein were consented participants in $\mathrm{NCl}$ Protocol 0I-C-0009, which was supported by support services contracts NO2-CP-I I019-50 and N02-CP65504-50 with Westat, Rockville, MD. We remain deeply grateful to the ongoing participation of the women who contributed their time and efforts to support this study.

\section{References}

I. National Center for Complementary and Alternative Medicine, Health Information [http://nccam.nih.gov/health/whatis $\mathrm{cam} /]$

2. Barnes PM, Powell-Griner E, McFann K, Nahin RL: Complementary and alternative medicine use among adults: United States, 2002. Adv Data 2004:1-19 [http://www.cdc.gov/nchs/data/ad/ ad343.pdf].

3. Eisenberg DM, Davis RB, Ettner SL, Appel S, Wilkey S, Van Rompay $M$, Kessler RC: Trends in alternative medicine use in the United States, 1990-1997: results of a follow-up national survey. JAMA 1998, 280( (18): 1569-I575.

4. Upchurch DM, Chyu L, Greendale GA, Utts J, Bair YA, Zhang G, Gold $E B$ : Complementary and alternative medicine use among American women: findings from The National Health Interview Survey, 2002. J Womens Health (Larchmt) 2007, 16(1):102-113.

5. Mao JJ, Farrar JT, Xie SX, Bowman MA, Armstrong K: Use of complementary and alternative medicine and prayer among a national sample of cancer survivors compared to other populations without cancer. Complement Ther Med 2007, 15:2I-29.

6. Richardson MMA, Sanders TT, Palmer JJL, Greisinger AA, Singletary SSE: Complementary/alternative medicine use in a comprehensive cancer center and the implications for oncology. J Clin Oncol 2000, I 8(13):2505.

7. Sparber A, Bauer L, Curt G, Eisenberg D, Levin T, Parks S, Steinberg SM, Wootton J: Use of complementary medicine by adult patients participating in cancer clinical trials. Oncol Nurs Forum 2000, 27(4):623-630.

8. Matthews AK, Sellergren SA, Huo D, List M, Fleming G: Complementary and alternative medicine use among breast cancer survivors. J Altern Complement Med 2007, 13(5):555-562.

9. Morris KT, Johnson N, Homer L, Walts D: A comparison of complementary therapy use between breast cancer patients and patients with other primary tumor sites. Am J Surg 2000, I79(5):407-4II.

10. Astin J, Reilly C, Perkins C, Child WL: Breast Cancer Patients' Perspectives on the Use of Complementary and Alternative Medicine: A Study by the Susan G. Komen Breast Cancer Foundation. Journal Soc Integrat Onc 2006, 4(4): 157-169.
II. Helyer LK, Chin S, Chui BK, Fitzgerald B, Verma S, Rakovitch E, Dranitsaris G, Clemons M: The use of complementary and alternative medicines among patients with locally advanced breast cancer - a descriptive study. BMC Cancer 2006, 6:39.

12. Rakovitch E, Pignol JP, Chartier C, Ezer M, Verma S, Dranitsaris G, Clemons $\mathrm{M}$ : Complementary and alternative medicine use is associated with an increased perception of breast cancer risk and death. Breast Cancer Res Treat 2005, 90(2): 139-148.

13. Montazeri A, Sajadian A, Ebrahimi M, Akbari ME: Depression and the use of complementary medicine among breast cancer patients. Support Care Cancer 2005, I 3(5):339-342.

14. DiGianni LM, Kim HT, Emmons K, Gelman R, Kalkbrenner KJ, Garber JE: Complementary Medicine Use among Women Enrolled in a Genetic Testing Program. Cancer Epidemiol Biomarkers Prev 2003, I 2(4):32I-326.

15. DiGianni LM, Rue M, Emmons K, Garber JE: Complementary Medicine Use before and I Year Following Genetic Testing for BRCAI/2 Mutations. Cancer Epidemiol Biomarkers Prev 2006, I5(I):70-75.

16. Emmons KM, Kalkbrenner KJ, Klar N, Light T, Schneider KA, Garber JE: Behavioral risk factors among women presenting for genetic testing. Cancer Epidemiol Biomarkers Prev 2000, 9(1):89-94.

17. Lerman C, Hughes C, Croyle RT, Main D, Durham C, Snyder C, Bonney A, Lynch JF, Narod SA, Lynch HT: Prophylactic surgery decisions and surveillance practices one year following BRCAI/2 testing. Prev Med 2000, 3 I (I):75-80.

18. Meiser B, Butow P, Barratt A, Friedlander M, Gattas M, Kirk J, Suthers G, Walpole I, Tucker K: Attitudes toward prophylactic oophorectomy and screening utilization in women at increased risk of developing hereditary breast/ovarian cancer. Gynecol Oncol 1999, 75(I): I22-129.

19. Meiser B, Butow P, Price M, Bennett B, Berry G, Tucker K: Attitudes to prophylactic surgery and chemoprevention in Australian women at increased risk for breast cancer. J Womens Health 2003, I 2(8):769-778.

20. Schwartz MD, Kaufman E, Peshkin BN, Isaacs C, Hughes C, DeMarco T, Finch C, Lerman C: Bilateral prophylactic oophorectomy and ovarian cancer screening following BRCAI/BRCA2 mutation testing. J Clin Oncol 2003, 2 I (2I):4034-404I.

21. Harris PF, Remington PL, Trentham-Dietz A, Allen Cl, Newcomb PA Prevalence and treatment of menopausal symptoms among breast cancer survivors. I Pain Symptom Manage 2002, 23(6):50I-509.

22. Newton KM, Buist DS, Keenan NL, Anderson LA, LaCroix AZ: Use of alternative therapies for menopause symptoms: results of a population-based survey. Obstet Gynecol 2002, 100(I):18-25.

23. Lerman C, Daly M, Masny A, Balshem A: Attitudes about genetic testing for breast-ovarian cancer susceptibility. J Clin Oncol 1994, I 2(4):843-850.

24. Lerman C, Trock B, Rimer BK, Jepson C, Brody D, Boyce A: Psychological side effects of breast cancer screening. Health Psychol I991, I0(4):259-267.

25. Radloff L: The CES-D scale: a self-report depression scale for research in the general population. Applied Psychological Measurement 1977, I:385-401.

26. Bono C, Ried, Douglas L, Kimberlin, Carole K, Vogel, Bruce : Missing data on the Center for Epidemiologic Studies Depression Scale: A comparison of $\mathbf{4}$ imputation techniques. Res Social Adm Pharm 2007, 3:I-27.

27. Narod SA, Offit K: Prevention and management of hereditary breast cancer. J Clin Oncol 2005, 23(8): 1656-1663.

28. Lee MM, Lin SS, Wrensch MR, Adler SR, Eisenberg D: Alternative Therapies Used by Women With Breast Cancer in Four Ethnic Populations. J Natl Cancer Inst 2000, 92(I):42-47.

29. Ashikaga T, Bosompra K, O'Brien P, Nelson L: Use of complimentary and alternative medicine by breast cancer patients: prevalence, patterns and communication with physicians. Supportive Care Cancer 2002, 10(7):542-548.

30. Boon $\mathrm{H}$, Olatunde $\mathrm{F}$, Zick S: Trends in complementary/alternative medicine use by breast cancer survivors: Comparing survey data from 1998 and 2005. BMC Womens Health 2007, 7(I):4.

3I. Helyer L, Chin S, Chui B, Fitzgerald B, Verma S, Rakovitch E, Dranitsaris $G$, Clemons $M$ : The use of complementary and alternative medicines among patients with locally advanced breast cancer - a descriptive study. BMC Cancer 2006, 6(I):39. 
32. Markman M: Safety issues in using complementary and alternative medicine. J Clin Oncol 2002, 20 ( 8 Suppl):39S-4 IS.

\section{Pre-publication history}

The pre-publication history for this paper can be accessed here:

http://www.biomedcentral.com/1472-6882/8/17/prepub

Publish with Bio Med Central and every scientist can read your work free of charge

"BioMed Central will be the most significant development for disseminating the results of biomedical research in our lifetime. " Sir Paul Nurse, Cancer Research UK

Your research papers will be:

- available free of charge to the entire biomedical community

- peer reviewed and published immediately upon acceptance

- cited in PubMed and archived on PubMed Central

- yours - you keep the copyright

Submit your manuscript here:

http://www.biomedcentral.com/info/publishing_adv.asp 